\title{
Novel Method of Management of Hyperbilirubinemia
}

\author{
Walter C Boutwell* \\ Neonatologist, Northwestern University, USA
}

*Corresponding author: Walter C Boutwell, Neonatologist, Northwestern

University, USA.

Received Date: October 09, 2020

Published Date: November 11, 2020

\section{Opinion}

The current management of hyperbilirubinemia in neonates is centered on determining levels at various ages and assigning management protocols based on zones as per the Bhutani curves, which are and remain extremely useful. This works well but has several deficiencies.

1) Bhutani curves are specific to never-treated infants. Once treated, either with phototherapy or with exchange transfusion, they are useless, as the curves were never created for that purpose.

2) The determination of a zone is based on "risk" factors is an all-or-nothing designation, making it difficult to deal with smaller or sicker patients.

Over several decades of dealing with hyperbilirubinemia, several aspects of the disease have been generally observed:

1) The concern has essentially nothing to do with jaundice, the incidence of which approaches universality, but rather kernicterus.

2) "Panic Levels" of bilirubin are reported irrespective of the age of the patient, the rate of rise of the bilirubin, or the amount of time to any expected intervention, such as an exchange transfusion.

3) Susceptibility of kernicterus is a threshold effect and not dose-responsive.
4) Exchange transfusion is rapid and successful in lowering bilirubin to safe levels

5) Exchange transfusions are associated with significant complications for the patient and extreme fatigue on the part of the practitioner.

6) Phototherapy's effect, creating trans-isomers that are more water-soluble, is very rapid, reversible, and clinically unmeasurable at the current time.

Several conclusions can be made from this:

1) Concerns are not based on the levels measured but rather on the speed with which the bilirubin concentration is increasing. "Slow stays low; fast goes high."

2) Phototherapy is not intended to prevent kernicterus but rather to prevent exchange transfusion.

3) The level of exchange transfusion can and should be adjusted individually for each patient based on the varying risk factors.

Therefore, it seems more reasonable to evaluate patients on how soon they will reach a level requiring exchange transfusion rather than evaluating them on the basis of how high their bilirubin is at any moment. While this has led to some tense discussion with medical and nursing colleagues who do not wish to consider exchange under any circumstances, it is in reality precisely what demands our consideration. If we are negligent or delayed in our 
use of phototherapy, the only alternative is exchange (now a rare intervention, performed by those whose experience is limited) $[1,2]$.

To avoid that eventuality, a few simple calculations may be performed that are not widely in use currently.

The first is specifying an exchange limit (EL) for each patient at the start of an evaluation. Obviously, for the term infant with no disease, the generally accepted limit of $25 \mathrm{mg} / \mathrm{dL}$ is used. While rare, kernicterus has been identified in healthy term infants. Lowering that limit based on the fragility of the patient, and disease states of the patient is beyond the scope of this piece but include birth weight, weight/PCA, PCA, acidosis, hyper/hypoglycemia, presence of infection, hypoalbuminemia, and asphyxia.

The second calculation is an estimate of the Rate of Rise (RoR) of the bilirubin. Even in the very most severely affected infants suffering from aggressive hemolytic disease, bilirubin at birth is remarkably low due to the efficiency of transplacental excretion. In most infants, an estimate of bilirubin at birth $\left(\mathrm{B}_{(0)}\right)$ to be equal to $1 \mathrm{mg} / \mathrm{dL}$ is reasonable unless the mother herself is jaundiced. Routinely obtaining a bilirubin level if an infant is noted to be jaundiced, or at $24 \mathrm{hrs}$ of age as is commonly done in most nurseries. Calculation of a presumed RoR is thus simple.

$$
\operatorname{RoR}=\left\{\left(\mathrm{B}_{(\mathrm{t})}-\mathrm{B}_{(0)}\right) / \mathrm{t}\right\} \times 24
$$

Where $(\mathrm{t})$ is the time difference in hours between the two determinations. This yields a normalized RoR in $\mathrm{mg} / \mathrm{dL} / 24$ hours. A simple calculation of the expected rate of rise in an infant who is merely hemolyzing senescent erythrocytes at a usual rate yields a RoR of 5.2-7.5 mg/dL/day, if there is no excretion whatever. Obviously, any value, even if obtained at an early age and in the absence of jaundice, exceeding this range, is presumptive evidence of increased heme destruction, whether intravascularly, due to ineffective erythropoiesis, or bruising. With each new bilirubin determination, a more accurate and reliable calculation of RoR can be calculated and compared to the previous determinations. Observing that the "rate of rise is going down" is surprisingly comforting to worried parents who only see an inexorable increase in bilirubin levels otherwise [3].

The next question to be answered is, "How long before we have to do an exchange." The days to exchange (DTE) is also a simple calculation:

$$
\mathrm{DTE}=\left(\mathrm{EL}-\mathrm{B}_{(\mathrm{t})}\right) / \text { RoR }
$$

where EL is the exchange level previously selected based on the patient's clinical condition, $\mathrm{B}(\mathrm{t})$ is the most recent bilirubin level, and RoR is the rate of rise just calculated.

This produces a single clinical estimate, which is uniform with all patients: the "Days to exchange," (DTE). The DTE is independent of the clinical state of the patient, the rate of rise, the presence of hemolysis, and the current bilirubin level. Few clinicians would fail to start phototherapy if the DTE were under 2. My personal limit is 2.5 days as a criterion to start intensive phototherapy.

In addition, this method operates whether the patient is currently under phototherapy or has previously been under such treatment. Once therapy is initiated, the RoR can be observed for changes suggestive of a clinical improvement. Stopping phototherapy too soon merely prolongs hospitalization when it must be restarted. If the RoR is shown to have an otherwise unexpected slowing, it can be surmised that excretion has improved, and discontinuation of treatment is reasonable.

This method has the added advantage of being extremely flexible, adjusting itself to any level of risk in which a patient presents. A simple flowsheet provides a common management strategy over time and with different practitioners.

Additional benefits accrue when one can be assured that the RoR is slowing (and an increasing DTE) when discharging a patient. Once enterohepatic circulation of bilirubin is discouraged by feedings and enteral excretion has increased (as demonstrated by a slowing RoR), the clinician can be confident that the patient can be discharged without repeated outpatient determinations of bilirubin, so frequently fraught with delay and uncertainty.

\section{Acknowledgement}

None.

\section{Conflict of Interest}

No conflict of interest

\section{References}

1. Jackson JC (1997) Adverse events associated with exchange transfusion in healthy and ill newborns. Pediatrics 99(5): E7.

2. Personal observation over a career which included the last of the $\mathrm{Rh}$ epidemic.

3. Maisels MJ, Newman TB (1995) Kernicterus in otherwise healthy, breast-fed term newborns. Pediatrics 96(4 Pt 1): 730-733.

Calculated as $\mathrm{H}$ (Hgb concentration in $\mathrm{g} / \mathrm{dL}$ ) $\mathrm{V}$ (blood volume/kg) x W( in $\mathrm{Kg}$ ) x 1/90 (fraction of RBCs removed each day due to normal senescence) $\mathrm{x}$ $35 \mathrm{mg} / \mathrm{G}$ of hemoglobin (bilirubin created in the metabolism of hemoglobin)/Vd (volume of distribution for a fat soluble moiety). On average, $\mathrm{H} \times \mathrm{V} \times \mathrm{W}$ x1/90 x 35mg/dL / W x V x \%Vd ( approximately unity) is 16G/dL x V x W x 35mg/dL/ $90=5.3$ to $7.6 \mathrm{mg} / \mathrm{dL} /$ day with a nominal average of $6 \mathrm{mg} / \mathrm{dL} / \mathrm{day}$. 